\title{
Clinical \\ Drug Investigation
}

\section{The Diagnosis and Treatment of Lower Urinary Tract Symptoms due to Benign Prostatic Hyperplasia: Primary Care Experience in Portugal}

\author{
Guest Editor \\ Matthias Oelke \\ Department of Urology, Hanover Medical School, \\ Hannover, Germany \\ Peer Reviewers \\ J. Kellogg Parsons, \\ Moores Comprehensive Cancer Center, \\ UC San Diego Health System \\ Francisco Gomez Veiga \\ Chairman of Urology Department and Kidney Transplant Unit, \\ Salamanca University Hospital, Salamanca, Spain
}

The supplement was supported by Tecnimede.

\footnotetext{
Conflicts of Interest The supplement was guest edited by Prof. Dr. med. Matthias Oelke who is a lecturer, advisor and/or study participant in the field of LUTS/BPH for Apogepha, Astellas, GlaxoSmithKline, Lilly, Mundipharma, Pfizer, and Recordati. He received a free yearly online subscription to the journal Clinical Drug Investigation.

The supplement was peer reviewed by Professor J. Kellogg Parsons who is lecturer at AMS, grant recipient and consultant at Watson Pharmaceuticals and consultant at Sophiris.

He received honoraria to peer review the supplement. The supplement was also peer reviewed by Prof. Francisco Gomez Veiga who didn't receive any honoraria and has no conflict of interest to declare.
} 